\title{
Seven Emergency Measures for Agricultural Vehicles Maintenance
}

\author{
WANG Yun
}

\author{
College of Mechanical Engineering , Baicheng Normal University, Baicheng,137000,China \\ email: 1306849091@qq.com
}

Keywords: Agricultural Vehicles, Cylinder Cushion, Jack

\begin{abstract}
In the course of agricultural vehicle driving, emergency measures can be used to carry out the repair work when the damaged parts cannot be repaired and the new parts can not be obtained. According to the previous working experience, this paper deals with seven emergency measures for the maintenance of agricultural vehicle from seven aspects, such as cylinder cushion burning out, radiator leakage, valve spring breaking, sudden power failure, clutch slipping, leaf spring breaking, and removing and replacing tires with no Jack, and is expected to provide some help to the relevant work.
\end{abstract}

\section{Introduction}

With the rapid development of agricultural mechanization in China, the number of farmers buying agricultural vehicles has risen sharply, which has played an important role in promoting the development of agriculture in China. After the use of agricultural vehicles for a period of time, various parts will appear wear or damage phenomenon, the use efficiency will be serious decline, failure factors will also increase, if serious, agricultural vehicles can not continue to use. Therefore, relevant users need to master the emergency maintenance skills of agricultural vehicles to avoid the impact of agricultural vehicle failures on working efficiency.

\section{Cylinder Cushion Burning out}

The cylinder cushion is between the cylinder head and the cylinder block, it can seal the cylinder of agricultural machine and avoid leakage of lubricating oil and cooling water after the cylinder head bolts are fixed. The main causes of cylinder head burning out are high temperature and high pressure gas shock, burn-out of package mouth and so on. When the cylinder head is burned out, there will be insufficient power and cylinder pressure, which will affect the normal use of agricultural vehicles.

The Cause of Cylinder Head Burning out. First, the cylinder head is prone to warp and deformation in the lower part, which causes the cylinder cushion to burn out. Second, during the installation of the cylinder cushion, because the cylinder head bolts are not tightened in the normal order, or because the torsion torque of the bolts is too large, the uncompacted part of the cylinder cushion is impacted by high pressure and cooling gas. As a result, the cylinder pad is damaged. Third, due to the insufficient height of the projecting part of the cylinder sleeve shoulder, the cylinder sleeve cannot be compacted into the engine block of the agricultural vehicle through the cylinder pad, thus making the high-pressure gas corrode the cylinder pad. In forth, the cylinder cushion quality of many agricultural vehicles itself does not meet the conditions, thus causing trouble easily. Generally speaking, the thickness of the cylinder cushion is about $2.5 \mathrm{~mm}$. During installation, the cylinder head bolts need to be tightened according to reasonable torque, but in many cases after the bolts are tightened, there will always be a small gap between the cylinder head and the upper end of the cylinder liner. If the cylinder cushion is made too thick, the gap will be further enlarged, which will cause the cylinder cushion to burn out under the impact of high temperature gas. In addition, in many agricultural vehicles, the difference in the thickness of the cylinder cushion is too large, and in the thin parts, it is difficult to ensure no leakage phenomenon [1].

The Cause of Cylinder Head Burning out. In the course of emergency maintenance, first of 
all, it is necessary to determine the specific location of the burning of the cylinder gasket. In order to achieve this goal, the agricultural vehicle driver can start the engine to ensure that the engine speed changes from low to medium speed, and then to high speed. If oil appears on one injector, the cylinder pad between the cylinder and the lubricating oil conveyer is damaged, which can be used after preliminary treatment. If the burning condition of the cylinder pad is not serious, the engine may appear the phenomenon of slow acceleration in the acceleration process, especially when the load of the agricultural vehicle is high, the whole body will appear obvious jitter, at this time, the driver should pay more attention and stop to check the cylinder pad to avoid serious burning of the cylinder pad. In addition, in the course of emergency maintenance, it is necessary to lock the locking parts according to the requirements, if there is negligence in this work, it may lead to serious consequences. For example, if the connecting rod bolt is not tightened or the tightening direction is wrong during maintenance, it may lead to the self-loosening of the engine connecting rod, which will lead to the phenomenon of tamping cylinder and make the engine being unable to continue to use. In addition, if the cylinder pad is burned to a lesser extent, it can be filled with substances such as asbestos wires carried with it; if the burned area is larger, a portion of the same spare cylinder pad can be used to fill it. The compactness must be guaranteed in the course of subsidy. If the damaged part is located between the two cylinders, it needs to be repaired by combining asbestos wire and copper skin to avoid the impact on the driving of agricultural vehicle [2].

\section{Radiator Leakage}

Radiator leakage is one of the frequent failures of agricultural vehicles. When the failure occurs, if the driver is unable to determine the leakage site at the first time, then the radiator should be removed and the upper and lower pipes of the radiator must be sealed, pump or irrigation method can be used to determine the location of the radiator leak at this time. The aeration method mentioned here is to immerse the radiator in the water and then use the pump to transfer air to the radiator. If there is any bubble coming out, the location of the leakage can be determined. When the inlet and outlet pipe of the radiator ruptures, it can be temporarily wrapped with tape or cotton cloth, and then wound around the periphery with wire and other substances. If the radiator ruptures more seriously, the driver can remove the ruptured hose and the ruptured segment, then connect the hose together using a tubular object, and then continue to use it after the wire is wound. When water leakage occurs in the upper and lower storage rooms of the radiator, it can be blocked by cotton or wood blocks of suitable size. If the water pipe of the radiator is leaking, it can be blocked with soapy soaps. When the leakage is very serious, the leakage can be forced to squeeze until no more leakage. In addition, in case of emergency, the glue can be bonded with related glue, such as 502 glue, and then the broken and leaky parts can be pressed by using the tools in agricultural vehicles. After pressing for a few minutes, the glue will play a role in bonding. Agricultural vehicles can be driven for some distance. However, these methods are mainly used in emergency situations. After the event, drivers should quickly seek repair workshops to avoid any impact on the subsequent use of agricultural vehicles [3].

\section{Valve Spring Breaking}

In the process of agricultural vehicle using, once the valve spring is broken, the proper parts can not be found for replacement. The following emergency measures can be used to remedy the situation. First, if the valve spring becomes very soft, a flat washer of about $2 \mathrm{~mm}$ thickness can be added under the spring and pressed at the bottom of the spring seat. After this operation, the effective increase of the spring pretightening force can be realized. Second, if the valve spring is broken, the driver can take the broken spring apart, and then pull the spring back together in a flat section, and lean the broken end against the spring base, so that, the valve spring can still function as before, but the effect and time will not last long. In addition, if there is only one broken part of the valve spring, it can be installed by a two-part spring, which can function as a basic valve spring. If the spring in 
the cylinder is broken more than one place, at this time the agricultural vehicle driver can remove all the bolts of the intake valve and exhaust valve of the cylinder so as to realize the integral closure of the valve. At this time the cylinder will stop working and the oil will be cut off. Thirdly, because the valve spring is broken, it is easy to cause the chain reaction of engine cylinder, even a series of problems such as top cylinder, valve break and so on, which will lead to engine scrapping in severe case. During the valve disassembly process, though it is urgent maintenance, attention must be paid to the accuracy of the disassembly and installation procedure. If the valve spring above the combustion chamber breaks, the load on the vehicle should be reduced after emergency repair. In the subsequent driving process, the driver can not step on the throttle frequently, otherwise the valve spring will easily appear loose and break again. In order to carry out the emergency repair of valve spring failure, the driver can separate backup, the valve spring in the process of agricultural vehicles using, in general, valve spring tractor is about $77 \mathrm{~mm}$. In addition, in the process of valve spring fracture emergency repairing, it is need to lock the lock valve spring seat, so as to provide the basic condition for the emergency repair of valve spring [4].

\section{Sudden Power Failure}

In the course of agricultural vehicle driving, the horn may suddenly not ring, the lights will all go out and the starter will stop turning, etc., which indicates that the agricultural vehicle has been completely cut off from electricity. At this point, the driver should first check whether the battery connector is loose. During this inspection, the other devices on the car must not be crowed at will. If there is no spark phenomenon, it can be explained that the reason of the failure is the loosening of the pile head. In this case, the driver only need to tighten it with his hand. If the phenomenon of power failure has not been restored after the tightening of the pile head, it is necessary to carefully check the connection of the two levels of conductors. If there is a phenomenon of poor contact, remedial measures should be taken in time. In addition, battery damage can also cause power failure, if it is a single damage, the driver can use copper wire to reconnect it. In many agricultural vehicles, glass hoists are installed in the process of use. This device is very serious to the loss of electric energy. However, because the battery and engine lines are not replaced for a long time, the lines may be damaged by overload. This can also lead to a blackout of the entire line. At this time, the driver can test the battery charge, if the car uses too much electricity, it can be turned off, and if the battery shows that there is electricity, the line can be fully tested, especially, increasing the attention in the connection mode and power matching degree [5].

\section{Clutch Slipping}

There are many reasons that cause the clutch to slip. For example, when there is oil on the friction plate, the function of the clutch will be affected. At this time, the driver of the agricultural vehicle can repeatedly trample on the clutch pedal and fill the oil gun with gasoline. Spray the gap of the flywheel, friction plate and other devices, can temporarily eliminate the oil stain in the friction sheet, if the oil gun can not be found for a time, it can also be replaced by plastic pipe and other tubular items. If the clutch is skidded because of the damage of the friction plate, the driver can coat the damaged friction piece to increase the friction force of the friction plate, and the mode clutch will continue to slip. But at this time, the agricultural vehicle must be stable in the starting process, and also need to change the speed in time and quickly, otherwise the clutch will continue to slip problem. In addition, when the spring between the friction plates is damaged and worn seriously, there will also be the problem of skidding due to the reduction of the effect between the friction plates. In this case, the driver can replace or deal with the spring of the friction disc for restoring his ability to work. The role of the clutch is to transfer the engine power to the drive shaft. If the clutch pedal is pressed down, the transmission will begin, and the transmission force is mainly realized by the friction of the clutch, if the agricultural vehicle is filled with oil, but still feel powerless when starting, it is necessary to pay attention to the clutch disc replacement at this time. In addition to the above, the driver can check the free stroke of the clutch pedal. If there is a problem with the free 
stroke, but the pedals can be raised completely, the length of the pull rod can be adjusted reasonably. If the clutch slip is not solved, the separation lever can be checked to avoid the phenomenon of excessive separation lever. If the normal clutch free travel, the driver needs to release bearing and sleeve for seizure detection, clutch cover and bolt is loose, if present, it should be immediately processed manually. If the friction plate appeared hardening and rivet leakage phenomenon, it should be replaced [6].

\section{Leaf Spring Breaking}

If the steel plate spring breaks in the course of driving of agricultural vehicles, then according to the actual situation, a small piece of wood with suitable specifications can be placed on the cushion between the axle and the frame, so as to make up for the deficiency of the broken spring, it is also need to use wire to bind it, and immediately go to the repair shop for repairs. Generally speaking, the breakage of individual leaf springs will not seriously affect the driving of vehicles, but if there are too many broken leaf springs, the remaining leaf springs will not be able to support huge loads, which will have an impact on the normal running of agricultural vehicles. At this time, the driver can also remove the assembly of the leaf spring, polish the two ends of the steel plate, and also use electric welding machine to surfacing, and then grinding the welding mouth with grinding wheel. If there is no welding condition, the side beam of the frame can be lifted up by means of Jack, and the broken part of the spring can be determined, and then fixed with the spring clip, so that the leaf spring can be temporarily restored. There are many reasons for the breakage of the leaf spring, the main reason is that the lubricating effect is decreased. During the use of agricultural vehicles, the staff should pay attention to the lubrication and maintenance of the internal frame, and in the course of emergency handling, It is also necessary to avoid large-scale friction between steel sheet and steel sheet, resulting in the mutual tension between leaf springs [7].

\section{Removing and Replacing Tires with no Jack}

In the use of agricultural vehicles, it is easy to ignore the Jack. If the tire is damaged without the Jack, the following emergency measures can be taken. If the outer wheel of double rows needs to be replaced, the driver can find the right piece of wood or stone and cushion it under the inner wheel, which can ensure that the outer wheel is suspended, thus facilitating the tire replacement work of the outer wheel. If the front wheel or single row tire is replaced, appropriate pieces of wood and stone can be placed under the front axle and rear axle of the agricultural vehicle, and the whole body will be kept in a stable state, after which earthwork will be carried out under the tyres that need to be replaced. Stop digging until the tire is suspended, so that the tire is replaced. In addition, a piece of wood perpendicular to each other at the top and side can be placed under the front axle and rear axle of the tire, while ensuring the smooth body of the body, the car can be started so that the block can fully lift the front axle or rear wheel. After the vertical block is smooth, the tire replacement can be carried out. If the double-row inner tire needs to be replaced, the inner and outer wheels can be unloaded together in the manner described above, the outer tire is mounted to the inside, and the appropriate block of wood is placed on the underside of the wheel, and the driver can then move the body forward. Make sure that the inner wheel falls on the stone or block and then installs the spare tire.

\section{Conclusion}

To sum up, agricultural vehicles play an important role in agricultural production. The maintenance work is not timely and the maintenance scope is not comprehensive, which leads to frequent failures in the use of agricultural vehicles. Therefore, in the use of agricultural vehicles, drivers need to master the emergency maintenance measures, once the failure occurs, the agricultural vehicles can continue to start through emergency treatment, avoiding the impact on agricultural work. 


\section{References}

[1] JIANG Lifen. Causes of excessive temperature of agricultural vehicle engine and its removal method[J].Friends of Farmers Becoming Rich,2016(03):109.

[2] MAO Liza·zhumahan. Application and maintenance of hydraulic system of Agricultural vehicle [J]. Agricultural Equipment \& Technology,2015,41(02):39.

[3] HUANG Jie. Disassembly and assembly of cylinder head assembly of diesel engine and inspection method for crack of cylinder head[J]. Agricultural Mechanization Using \& Maintenance, 2015(01):80.

[4] CHEN Yi, LEI Changhao, HAO Ye. Research on fault caused by throttle of agricultural vehicle engine[J].Science \& Technology Vision,2014(33):23+114.

[5] HUANG Guohua. Discussion on concrete construction technology in spillway reconstruction project[J]. Heilongjiang Hydraulic Science and Technology,2014,42(04):143-144.

[6] CHEN Ye. Discussion on Green maintenance and Scientific Management of Agricultural vehicles[J]. Beijing Agriculture,2014(12):188.

[7] LI Yong. The cause of excessive oil consumption and the way to reduce costs of diesel engine[J]. Agricultural Mechanization Using \& Maintenance,2014(04):17. 